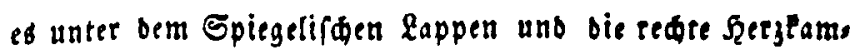
mer gebalten wurbe; als bet Sepf gedfnet worden und bas Thermometer in bie Şirnfubftanz gebradte war, fiel es fo, gleid anf $82^{\circ} \mathrm{f}$.

10) Fin Mann yon 28 Jabren ftarb an einer Reber, Pranlbeit. Die @eide waro bei einer Remperatur von $58-$ $60^{\circ}$ unlerfudt. Ein in bie Şirnfubftanz gebradtes Thers mometer fieg auf $7^{\circ}$; etroa eine balbe Stunde fpater ftieg es unter ber redien f̧erjlammer und unter bet \&eber auf $32 \mathfrak{F}$.

So weit bie Beobadtungen beb $\mathfrak{B e t f}$, die tvir fo aus, fübrlid als móglid bier mitgetheilt haben. Befonders bes merpenswerth erfdien es uns, dá in den Fällen bes acuten Rheumatisinus unb ber Apoplesie ber 23 drmegrab unter bem Feerzen uno der leber fo beoeuteno grop war; eben fo, Daßs bas f̧irn ftetó einen ungleid geringeren $\mathfrak{B a r m e g r a d}$ ald bas $\mathfrak{F}_{2} \mathrm{er}_{3}$ und bie feber hatte.

\title{
Ueber Ferrum carbonicum;
}

von

S. Filix,

5and. Der Pharmacie der zeit in Marburg.

Dad in neuern Beiten officinell geworbene tủnflide Publenfaure cifenoryoul lafit fid nad ben bisber yorbandes nen Borfdriften ourdaub nide troden barfellen, fonbern wathreno bem Trotfnen beim Zutritt ber luft (10elde $\mathfrak{B a}=$ me mall aud anwenoen $\mathrm{mag}$ ) verwandelt fic ber grsate Theil in Eifenoxydgyorat. Selbft das nad $B \mathfrak{B}$ d ner's Retbobe (Repert. Bd. 28. S. 397) bereitete toblenlaure Eifen enthått taum die Scalfte Roblenfåure, als twenn bafs felbe im luftleeren, oder ood wenigftens luftuerbinnten 
Raume getrodnet wirb. Mein Berfabren tft folgende6:

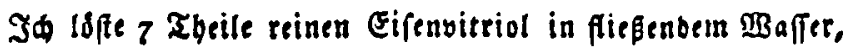
uno da bie fssung von toblenfaurem Pali nidt gand rei gefallt wurbe, todere id fie nad einiger Zeit mit metallis f由em Eifen, und filtritte fie fanell in ein bohes mit einem Etspfel gut zu verfdließended Enlinberglas, feşte alsbann 4 Theile Kali carb. dep. iu ausgefodtem nod heibem beft. IBaffer gelsft, bingu, füllte die Flafめe ganz mit allsger Podrem $\mathfrak{B a f f e r}$ an, uno ftelle fie feft verftopft jum 2 bfetzen bei Eeite. Nadbem fid der graulidineife Nieberfdlag ges reßzt hatte, gob i由 oab Szelle $a b$, füllte bie Flafde wieber

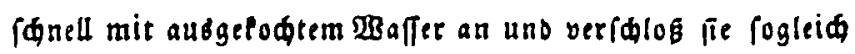
wieber. Diefes warbe nod einigemal wieberbolt, bis bie

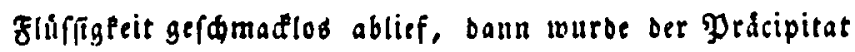
nod einmal mit 2lltobol allggewaften, nadbem er fí wieber abgelagert, ber baruber flebende difobol abgegoffen, uno ber breiartige Riederfílag fo fhnell ald mojglid in ein Eleines tubutirtes Retsrtden gegoffen. Borber mub foou eine etwas grope Borlage angepart fenn, am beften cine ftarfe Flafde mit engem Salle, woourd ein ourdbobrter Furt geffect wirb. Die Flafde wirb mittelft etwas $\mathbb{B a f f e r}$, wel, des faft bid jum Sieden erhiket wirb, oder beffer ourd 2In, junben yon etwas $\mathfrak{B e i n g e i f t , ~ f o ~ v i c l ~ a l s ~ m i g l i d ~ l u f t l e e r ~ g e s ~}$ madt (Sollte nidt, um bie atmosphárifde \&uft tregzus

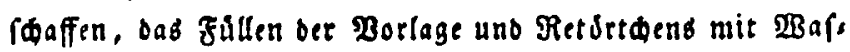
ferftoffgab oder Soblenfåure zwed máig feun?), bann (a) nelf Das Returtben feft bineingeffect und zum Ueberfú num verfittet. Nacbem bie Borlage talt geworben, wirb bas Retsrtwen ourd wenige Soblen erwdrmt, wo benn ber leşte

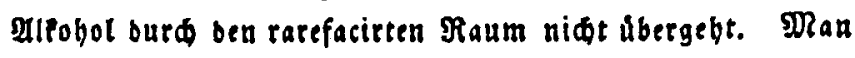
bute fid.aber ja, ftarl za erbigen, weil es fonft oie Siobs lenfáare fabren (ât, woburd leide ber Apparat zerfprengt werben esunte. Die Borlage wirb befandig talt gebalten, 
uno das Eriodrmen bes Retdrtdens fo lange fortgefest, bis Der Jnbalt vollig troden ift.

Dad auf oiefe Art erbaltene Pohlenfaure Eifenorybul

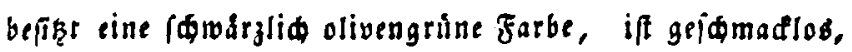
yon ben Sauren, befonder ber Salzfare, wiro eb leidt unter febr flarpem Rufbrauren gelsft. In jo Jiran fanb id 9 (3) ran Sublenfåure, wäbreno oas nad $\mathfrak{B}$ ud ner's Mes thode erbaltene nur etwas iber 4 (bran Robleufare enthielt. In ber \&uft bebalt es lange feine Farbe und yermandelt fid nur langlam uno theilweife in Fifenornobobrat um, was jebod bei äufaßz von $\mathfrak{B a f f e r}$ viel jđneller gefdiebt, baber es gut verflopft aufbewagre twerben mus.

2lis biefes ldon zum abfenden bereit war, fand id im Seprember, freit von (B) eiger'd Magazin, oaß Fere 2lpos theter Dr. Moloengaver in Franfurt a. o. Soer ein Búnflic roblenfaures Eifenojyoul von grünlid weiser Farbe burd 2lustrofnen unter der \&uftpumpe bereitet babe.

Da biefe Methode fúr oie Mharmaceuten von befdranfterm praftifकen $2 B$ erthe ift, weil bie Ruftpumpe ein febe toftba, res Inftrument iff, bas tur wenigen zu Bebote ftebt, fo glaube id nod obige sethode befanut madan za esunen.

Nod) eine Bemerlung úber Kali bicarbonicum; bon Ebendemfelben.

Unter allen Burføriften zur Boreitung eines Kali bicarb. foeint mir bie yon $\mathcal{S}_{\text {ebl }}$ meyer (Kastner's Archiv II. S. 495) fúr ben 2atpotbeter bie vortheilbaftefte zu feyn, indem er baffelbe bei Bereitung bes Tart. tart. als Rebens probuft erbalten tann. Danethişt ju dem Enoe tine gerei, nigte concentritte $\mathfrak{P}$ ottaf由enlofung bio obngefábr auf $60^{\circ} \Re$. , uno fattigt fie fo lange mit Tart. dep. bis bie $\mathcal{L}_{a}$ ge ftart zu 